\title{
Anomalía coronaria sospechada por el signo "RAC" en Resonancia Magnética Cardíaca
}

\author{
Julián Vega ${ }^{1}$, Javier López-Opitz ${ }^{1}$, Hugo Martínez ${ }^{1}$, Daniel Lorenzatti ${ }^{1}$, Javier Urmeneta ${ }^{1}$, Diego Lowenstein ${ }^{1}$. \\ 1. Cardiólogo especialista en imagen cardíaca, Instituto Chileno de Imagen Cardíaca INCIC www.incic.org.
}

\section{Anomalous coronary artery suspected by cardiac magnetic resonance "RAC" sign}

A 63 year-old-male was admitted with a non-ST-segment elevation acute coronary syndrome. Due to poor acustic windows ventricular function could not be evaluated by echocardiography. Magnetic resonance (CMR) revealed normal biventricular size and function without fibrosis and portraying a rare finding consisting of a tubular structure originated in the aortic root following a retro aortic course between the aorta and left atrium, traversing through the atrioventricular groove,. These characteristics raised the suspicionn of an anomalous coronary artery origin. Coronary angiography confirmed the pre- sence of two coronary arteries, the right coronary artery (RCA) and the left circumflex artery (LCx) originating from the right coronary sinus via two separate ostia. The LCx followed a retro aortic course proximally before irrigating the left ventricular lateral wall. From the left coronary sinus, the left anterior descending artery followed its normal course with a thin diagonal vessel that presented an acute plaque. Due to these low-risk findings, medical management was chosen.

Keywords: Coronary Vessel Anomalies, Coronary Angiography, Magnetic Resonance Angiography. 


\section{Caso:}

Hombre de 63 años, fumador activo, sin otros factores de riesgo cardiovascular, ingresado por un síndrome coronario sin elevación del segmento ST. Evoluciona estable, libre de dolor, por lo cual se programa coronariografía para el día siguiente. La ecocardiografía transtorácica no permitió valorar la función segmentaria debido a malas ventanas acústicas, efectuando el mismo día del ingreso una resonancia magnética cardíaca (RMC) en un sistema de 1.5 Tesla (Ingenia, Philips, Best, The Netherlands). Se demostró función y tamaño biventricular normal sin áreas de fibrosis miocárdica ni infarto. La secuencia de CINE (b-SSFP) de 4 cámaras del corazón resultó ligeramente desplazada hacia anterior (más próxima a la aorta), registrando un curioso hallazgo, consistente en una estructura tubular de interior hiperintenso y bordes finos e hipointensos, proveniente de la raíz aórtica, con un curso entre la aorta y la aurícula izquierda y siguiendo el trayecto del surco auriculo-ventricular (Figura 1, Panel A-B). Por sus características se sospechó que correspondiese a una estructura vascular de localización atípica, posiblemente una arteria coronaria con origen y trayecto anómalo. La curva de troponina resulta negativa, catalogando el cuadro como angina inestable. Dada la tipicidad del dolor se realiza una coronariografía vía arteria radial, confirmando la sospecha de la RMC, identificando en el seno de Valsalva derecho el nacimiento independiente (dos ostia) de dos arterias coronarias, la arteria coronaria derecha (ACD) con trayecto habitual y la arteria circunfleja (ACx), esta última con un curso retroaórtico ("RAC") para luego dirigirse hacia la cara lateral del ventrículo izquierdo (Figura 2, Panel A). La arteria descendente anterior (DA), tenía su origen de forma independiente desde el seno de Valsalva izquierdo, siguiendo su curso habitual por el surco interventricular y presentando una lesión aguda no obstructiva en una muy fina y pequeña rama diagonal, que se dejó en manejo médico. Debido a que la anomalía coronaria descrita corresponde a una variante de bajo riesgo y sin potencial de producir isquemia no se realizaron otras pruebas diagnósticas y el paciente quedó con terapia de prevención secundaria cardiovascular.

\section{Discusión:}

Las anomalías de las arterias coronarias son infrecuentes, encontrándose en aproximadamente el $1 \%$ de los pacientes referidos para coronariografía invasiva ${ }^{1}$. Dependiendo del origen y curso proximal pueden tener riesgo de compresión entre estructuras vasculares y/o cardíacas. El origen de la ACx desde el seno de Valsalva derecho, descrito por primera vez en 1933 por Antopol y Kugle ${ }^{2}$, es la anomalía coronaria más frecuente y de menor riesgo pues no tiene peligro de compresión ni pinzamiento. Respecto a su origen en el seno de Valsalva derecho, este puede ocurrir desde un ostium independiente, un ostium compartido

Figura 1. Hallazgos en la resonancia magnética cardíaca

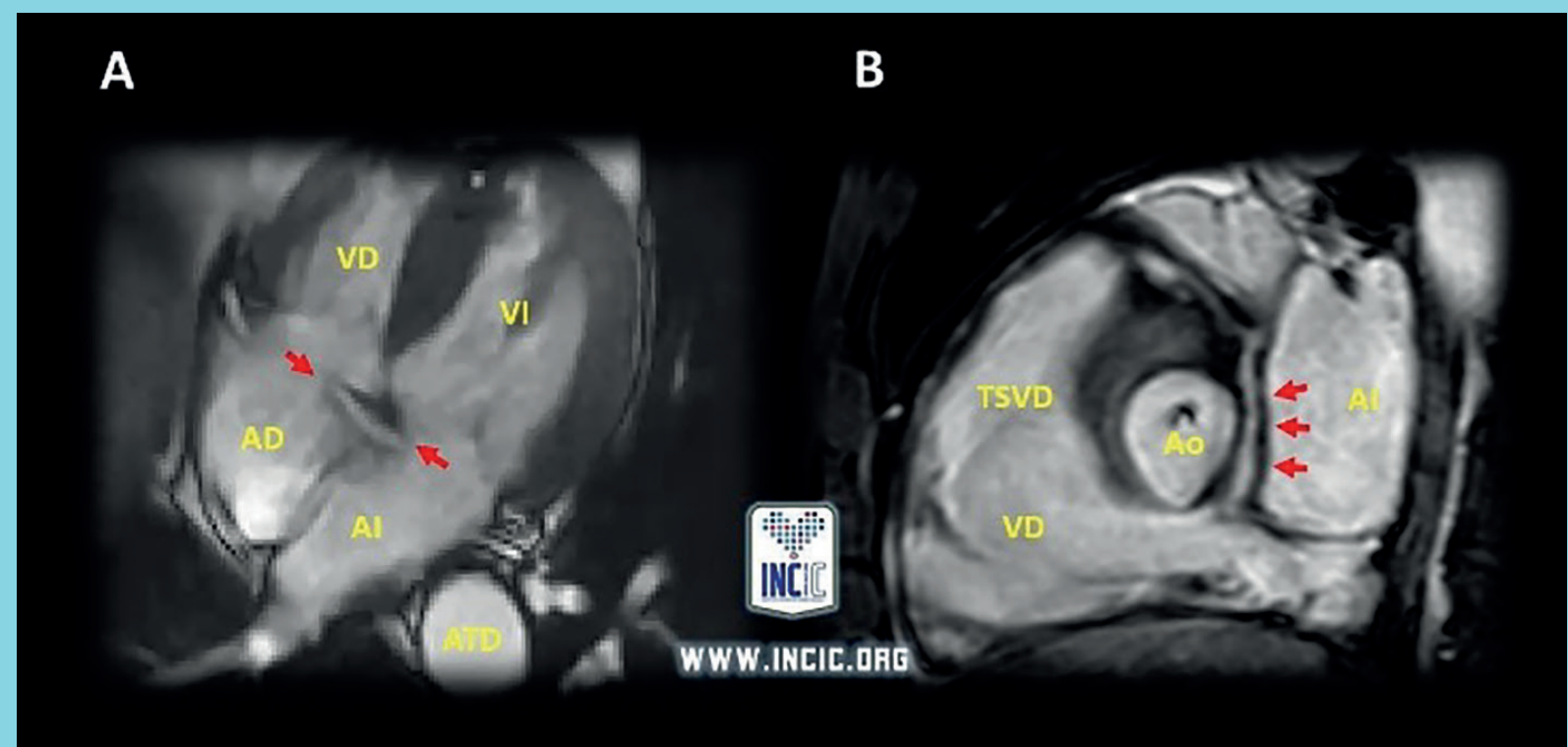

Panel A: CINE de 4 cámaras del corazón, se observa la estructura tubular descrita señalada por las flechas de color. Panel B: CINE con una vista de 3 cámaras del ventrículo derecho modificada que muestra dicha estructura tubular con un trayecto retroaórtico y anterior a la auricular izquierda. AD: Aurícula derecha, AI: Aurícula izquierda, Ao: Aorta, ATD: Aorta torácica descendente, VD: Ventrículo derecho, VI: Ventrículo izquierdo. 
Figura 2. Cateterismo coronario e ilustración anatómica.

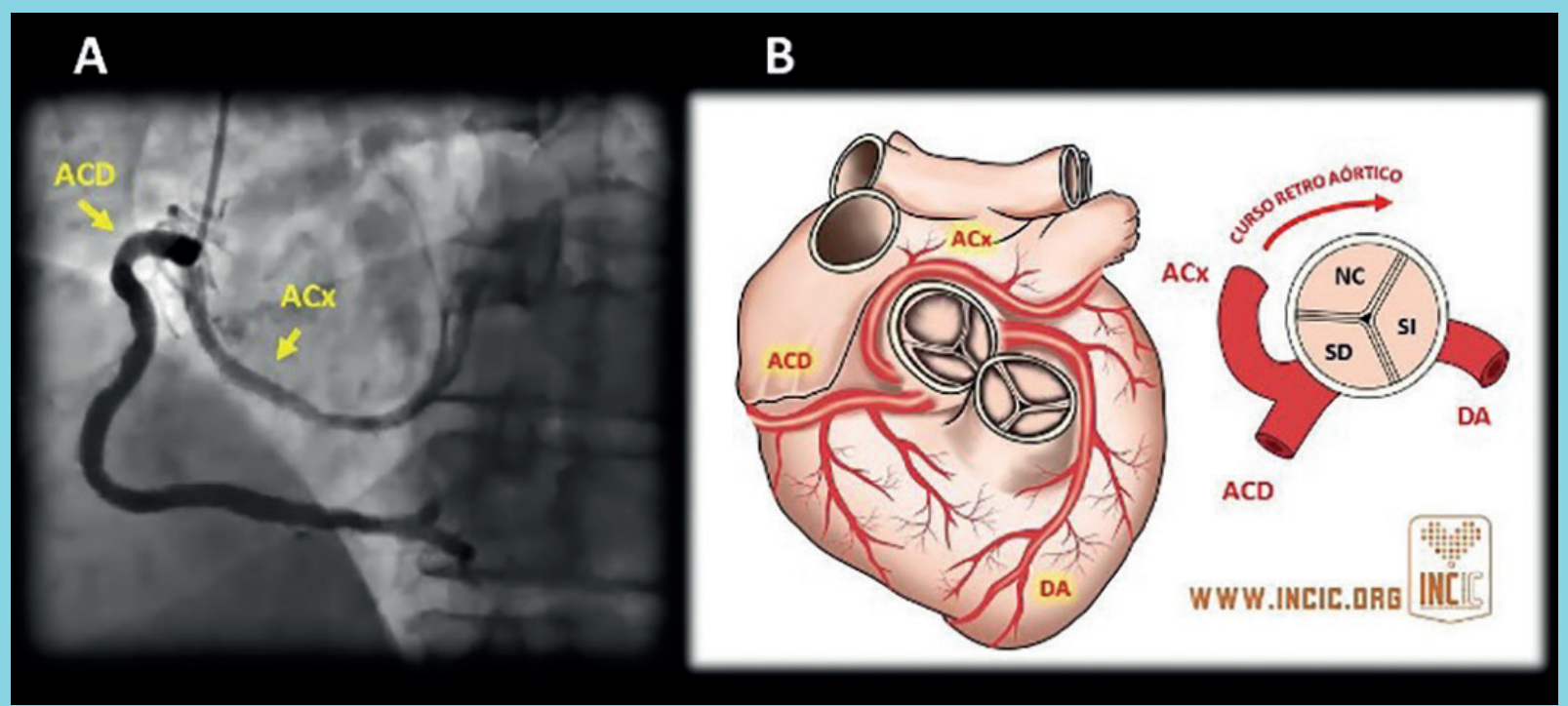

Panel A: Coronariografía, visión oblicua anterior izquierda que muestra el origen independiente de la arteria coronaria derecha (ACD) y la arteria circunfleja $(A C x)$ desde el seno de Valsalva derecho.

Panel B: Ilustración que demuestra la distribución de las arterias coronarias. Se observa el origen de la ACx desde el seno de Valsalva derecho (SD) siguiendo un curso retroaórtico para dirigirse a la cara lateral del ventrículo izquierdo. La arteria descendente anterior (ADA) se origina de forma solitaria desde el seno de Valsalva izquierdo (SI).

ACD: Arteria coronaria derecha, ACx: Arteria circunfleja, DA: Descendente anterior, NC: Seno no coronario, SD: Seno coronario derecho, SI: Seno coronario izquierdo.

con la ACD o presentar su origen desde la ACD proximal como una rama de esta.

Independiente de las características de su origen, su curso siempre es retroaórtico ("RAC") para luego ingresar al surco auriculoventricular izquierdo e irrigar los segmentos miocárdicos habituales (Figura 2, Panel B). El curso proximal retroaórtico es el responsable del llamado signo "RAC" (retroaortic anomalous coronary) en la literatura anglosajona, que consiste en visualizar una estructura tubular de aspecto vascular originada en el seno de Valsalva derecho que sigue un trayecto retroaórtico y transcurre posterior a la aurícula izquierda hacia la cara lateral del ventrículo izquierdo. Este signo "RAC" ha sido reportado en Ecocardiografía Doppler de superficie con creciente frecuencia, pero con una tasa de falsos positivos cercana al $6 \%^{3}$, dada principalmente por la presencia de artefactos en el ultrasonido. Por el contrario, el hallazgo del signo "RAC" por RMC, escasamente reportado en la literatura, tendría mucho mayor confiabilidad al estar libre de artefactos y permitir visualizar cortes anatómicos sin restricción. Finalmente, es relevante mencionar que existen secuencias avanzadas de angiografía coronaria por RMC - no disponibles en el equipo al momento de adquirir las imágenes en este caso - que permiten evaluar con una sola técnica ("one-stop-shop") toda la información anatómica requerida en las anomalías coronarias. Más aún, con el desarrollo de nuevas técnicas de flujo 4D por RMC es posible incluso obtener curvas confiables de flujo intracoronario. Todo esto posiciona a la RNM cardíaca, en el futuro cercano, como una atractiva técnica para el estudio de las alteraciones coronarias".

\section{Referencias}

1. YAMANAKA, O., HOBBS, R. E. Coronary artery anomalies in 126,595 patients undergoing coronary arteriography. Catheterization and Cardiovascular Diagnosis, 1990, 21(1):28-40.

2. ANTOPOL, W., KUGEL, M. A. Anomalous origin of the left circumflex coronary artery.
American Heart Journal, 1933, 8(6): 802-806.

3. WITT, C. M., ELVERT, L. A., KONIK, E. A., AMMASH, N. M., FOLEY, D. A., FOLEY, T. A. The RAC Sign. JACC: Cardiovascular Imaging, 2018, 11(4): 648-649. 